\title{
Bound polarons in semiconductor nanostructures
}

\author{
U. Woggon* and D. Miller \\ Experimentelle Physik IIb, Universität Dortmund, Otto-Hahn Strasse 4, D-44221 Dortmund \\ F. Kalina and B. Gerlach \\ Theoretische Physik IIb, Universität Dortmund, Otto-Hahn Strasse 4, D-44221 Dortmund, Germany \\ D. Kayser, K. Leonardi, and D. Hommel \\ Institut für Festkörperphysik, Universität Bremen, Kufsteiner Strasse 1, D-28359 Bremen, Germany
}

(Received 19 September 2002; published 27 January 2003)

\begin{abstract}
Bound polarons are discrete, confined electronic states, spatially localized due to a local potential $V(r)$ but sharing a common phonon state of the surrounding crystal. We study the energy states of polarons bound in a potential and determine the local optical absorption spectrum up to first-order time-dependent perturbation theory with respect to the electron-photon interaction. The model is applied to describe the optical properties of submonolayer CdSe insertions epitaxially grown between ZnSe layers. As a typical signature of bound polarons we found excited-state energies equidistantly separated by the LO phonon energy and with optical transition probabilities determined by the anisotropies in $V(r)$.
\end{abstract}

DOI: $10.1103 /$ PhysRevB.67.045204

PACS number(s): 73.22.-f, 71.38.-k, 78.67.-n

The present semiconductor technology provides artificial nanostructures of smallest length scales. To understand the optical and electronic properties of such engineered semiconductors, concepts of quantum confinement are well established. Nowadays, the modern semiconductor epitaxy even allows us to create ultrathin layers with submonolayer coverages. ${ }^{1}$ The discussion of the electronic and optical properties of such quantum structures is presently dominated by quantum dot concepts, i.e., pure electronic quantum confinement created by a potential barrier due to the surrounding semiconductor material. Such an approach seems to be justified since the formation of nanometer-sized islands of $\mathrm{CdSe}$ is proven by high-resolution electron microscopy, e.g., in case of $0.7-\mathrm{ML}$ (monolayer) deposition on $\mathrm{ZnSe}^{2}$ Current semiconductor technology, however, is able to manufacture nanoscale structures even by submonolayer growth with only $10-20 \%$ nominal surface coverage. Such structures are excellent candidates to study excitons in potentials that are either formed or influenced by local lattice distortions. Submonolayer II-VI nanostructures are highly polar materials with considerable exciton-phonon coupling, and the question arises: Is a "quantum dot" characterized by a pure electronic confinement still the correct picture to describe localized electronic states of nano-objects within a polar crystalline lattice?

In this work we present a bound-polaron concept to describe the confined states of a quantum structure with submonolayer insertions $(<0.5 \mathrm{ML})$. The polaron in a potential, i.e., the bound polaron, is considered of essential importance to understand nanostructures grown at atomic length scales: It refines the quantum confinement models if the electron-phonon interaction becomes important and introduces localized electronic states that couple to a common phonon state. The particular optical properties lead to an absorption spectrum that strongly deviates from the quantum dot or quantum well absorption caused by pure electronic confinement. In a bound-polaron picture, peaks separated by $\hbar \omega_{\text {LO }}$ in the absorption spectrum are explained in terms of eigenstates of the system. Such a picture has implications for the understanding of coherence in semiconductor nanostructures while relaxation processes would be described here as a simple polaron decay with different lifetimes for ground and excited polaron states, i.e., in terms of a polaron-polaron interaction process. In semiconductors, the bound polaron was until now merely of academic interest. Because of the technological progress in submonolayer growth, such elementary excitations can now be studied in real nanostructures.

The samples studied are grown in migration-enhanced epitaxy by depositing nominal 0.15 (0.58) ML's of CdSe on $\mathrm{ZnSe}$, i.e., we approach the limit of very low CdSe deposition. The growth of such submonolayer structures introduces a controlled local lattice modification, e.g., in a $\mathrm{CdSe} / \mathrm{ZnSe}$ quantum structure the $\mathrm{Zn}$ atoms in the $\mathrm{ZnSe}$ lattice are exchanged against $\mathrm{Cd}$ atoms. The number of $\mathrm{Cd}$ atoms is, however, much smaller than in self-organized grown islands, and not homogeneously distributed like in $\mathrm{ZnCdSe}$ mixed crystals. The sample scheme is shown in the inset of Fig. 1. First, a $\mathrm{ZnSe}$ buffer layer is grown on $4^{\circ}$ misoriented $\mathrm{GaAs}(001)$ substrates. Then three submonolayers of $\mathrm{CdSe}$ are grown between $\mathrm{ZnSe}$ layers finally capped by a ZnSe layer. ${ }^{3}$ The host material ZnSe has a band-gap energy of $E^{\mathrm{gap}}=2.82 \mathrm{eV}(T$ $=4 \mathrm{~K})$, an exciton binding energy of $19 \mathrm{meV}$, and an LOphonon energy of $31.5 \mathrm{meV}$. For the Fröhlich coupling strength of $\mathrm{ZnSe}$, we use $\alpha=0.432$. The photoluminescence (PL) is measured under optical excitation by a Xe lamp ( $T$ $=6 \mathrm{~K})$ and plotted in Fig. 1 . The emission maximum is at $2.7917 \mathrm{eV}(2.764 \mathrm{eV})$ for the 0.15 ML (0.58 ML) sample (for a survey of emission energies for different ML thickness, see Ref. 4). The PL spectrum is dominated by only one sharp peak with a full width at half maximum of $2 \mathrm{meV}$ for the 0.15-ML and $6.6 \mathrm{meV}$ for the 0.58-ML sample.

The photoluminescence excitation (PLE) spectrum is obtained by detecting the emission intensity at a fixed spectral position (here the maximum of the PL spectrum), while the 


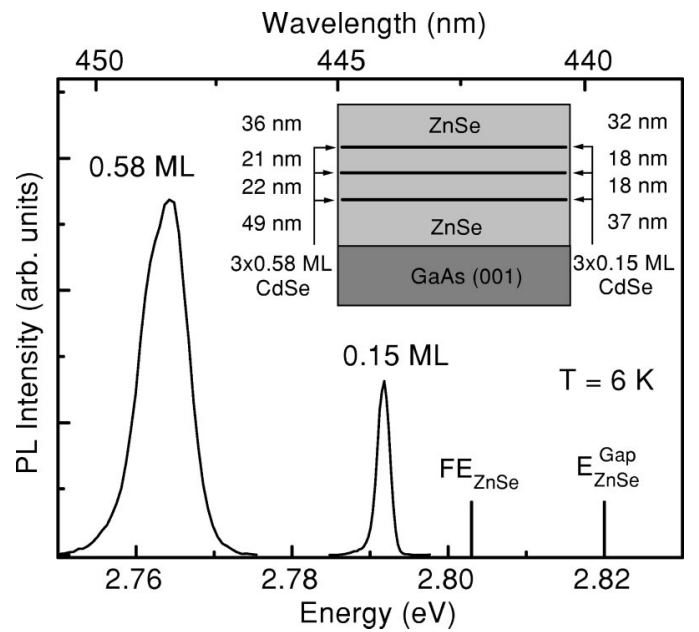

FIG. 1. PL spectrum of $\mathrm{CdSe} / \mathrm{ZnSe}$ quantum structures with 0.15-ML and 0.58-ML insertions of CdSe in ZnSe. The sample structure is shown in the inset. For comparison, the energy of the free exciton denoted by $\mathrm{FE}$ and of the band-gap energy $E^{\mathrm{Gap}}$ of bulk $\mathrm{ZnSe}$ is indicated.

energy of the exciting light source is spectrally tuned from 2.77 to $3.1 \mathrm{eV}$ by spectrally dispersing the light of a highpressure xenon lamp $(150 \mathrm{~W})$ by a $1-\mathrm{m}$ monochromator. The result is shown in Fig. 2. We observe in PLE up to ten equidistant peaks separated by the LO-phonon energy even at energies high above the $\mathrm{ZnSe}$ band edge. The peak heights and line shapes are extremely sensitive to the monolayer coverage and deviate from the expected Poissonian intensity distribution for the given phonon coupling constant $\alpha$. For the 0.15-ML sample, e.g., the second peak in PLE is the strongest, but for the 0.58-ML one, the first is dominating. While the PLE spectrum shows very pronounced peaks, the PL spectrum is dominated by the single, spectrally narrow peak used for PLE detection. The LO-phonon replica in the region below the PL maximum are by a factor of $10^{2}-10^{4}$

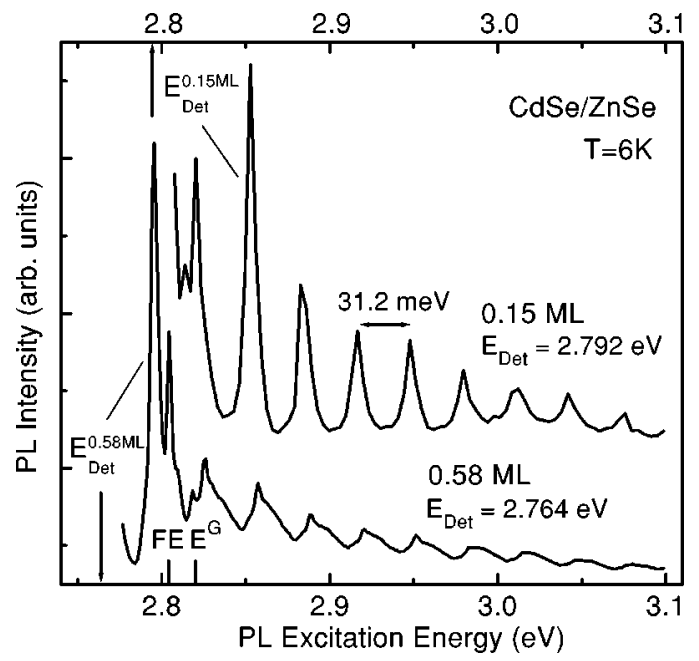

FIG. 2. PLE spectrum of the sample shown in Fig. 1 measured at $T=6 \mathrm{~K}$ with the detection energy tuned into the PL maximum. The peak energy separations are equal to the $\mathrm{ZnSe}$ LO-phonon energy. weaker in their emission intensities. The latter findings are in clear contradiction with the concept of a constant, material specific Huang-Rhys-Parameter $S$.

To explain the observed spectrum, we propose a boundpolaron concept that is based on the true eigenstates of the exciton-phonon system. The theoretical treatment is based on the functional-integral method and makes use of the following fact: If $E$ is an eigenvalue of the bound-polaron problem, the same holds for $E+E_{\mathrm{LO}}$, where $E_{\mathrm{LO}}$ is the LO-phonon energy. The observed peak energies in the excitation and emission spectra can nicely be explained that way. Since we have no direct access to the explicit wave functions using this exact treatment of the Hamiltonian, line shapes and exact peak intensities cannot be described here. In the frame of a model calculation we can show, however, the sensitivity of optical transition probabilities to the potential properties, e.g., its anisotropy.

Polaron formation has been discussed for bound-exciton complexes in $\mathrm{CdS}$ in an early paper, ${ }^{5}$ for $\mathrm{CuCl}$ nanocrystals, ${ }^{6}$ and very recently in InAs self-assembled quantum dots. ${ }^{7,8}$ Conceptually, Ref. 8 stresses the necessity to use a polaron concept for an adequate description of exciton-phonon phenomena in quantum dots, as we do here. The authors perform a variational calculation for the lower part of the spectrum, taking up to two-phonon states into account. We are concerned with higher excited states, thereby complementing the discussion in Ref. 8. We calculate transition energies for one polaron in a potential $V(r)$ that is subjected to a radiation field. Since the experimental spectra are clearly two-polaron (namely, exciton) data, the presented theoretical discussion accounts for the center-of-mass part of the bound exciton. It can directly be transferred to the complete exciton-polaron system, as has been examined in detail by us and will be presented in a forthcoming publication. The main conclusions with respect to the energy spectrum are unaffected using this approach.

The polaron in an arbitrary potential $V(r)$ can be described by a Hamiltonian of general type,

$$
\begin{aligned}
H_{P}= & \sum_{n} \varepsilon_{n} a_{n}^{+} a_{n}+\sum_{q} \hbar \omega(q) b_{q}^{+} b_{q} \\
& +\sum_{q n_{1} n_{2}} g_{q} M_{q n_{1} n_{2}} b_{q} a_{n_{1}}^{+} a_{n_{2}}+\text { H.c. }
\end{aligned}
$$

Here, $q, b_{q}, b_{q}^{+}, \omega(q)$, and $g_{q}$ are wave vector, annihilation and creation operators, dispersion, and coupling of the phonon branch under consideration; $g_{q}$ is the standard Fröhlich coupling. ${ }^{9,10}$ The matrix elements

$$
M_{q n_{1} n_{2}}:=\int d^{3} r e^{i q \cdot r} \Psi_{n_{1}}^{*}(r) \Psi_{n_{2}}(r)
$$

contain the solutions of the eigenvalue problem

$$
\left[\frac{p^{2}}{2 m}+V(r)\right] \Psi_{n}(r)=\varepsilon_{n} \Psi_{n}(r) .
$$

Here $p, r, n, m, a_{n}$, and $a_{n}^{+}$are momentum operator, position operator, quantum number, mass, and annihilation and cre- 
ation operators of the particle of interest; $V(r)$ denotes an effective potential that confines the particle.

If we restrict ourselves to the subspace of one-particle states, an arbitrary eigenstate of $H_{P}$ has the general form

$$
|\Phi\rangle=\sum_{N=0}^{\infty} \sum_{n q_{1} \cdots q_{N}} c_{n q_{1} \cdots q_{N}} a_{n}^{+} b_{q_{1}}^{+} \cdots b_{q_{N}}^{+}|0\rangle,
$$

where $|0\rangle$ denotes the Fock vacuum. In view of the Bose character of the phonons, the coefficients $c_{n q_{1} \cdots q_{N}}$ have to be an infinite sequence of totally symmetric functions of the wave vectors $q_{1} \cdots q_{N}$. It proves useful to extract the couplings from the unknown coefficients $c$ and to incorporate the symmetry conditions explicitly. This is done by the replacement

$$
c_{n q_{1} \cdots q_{N}}=\frac{(-1)^{N}}{N !} \prod_{\nu=1}^{N} g_{q_{\nu}}^{*} \sum_{\left.q_{1} \cdots q_{N}\right)} e_{n q_{1} \cdots q_{N}} .
$$

Here, $P$ indicates a permutation of all momenta $q_{1} \cdots q_{N}$; the coefficient $e$ is free of any symmetry requirements. In Ref. 11, the preceding substitution has been introduced to analyze the Fock-space equations of a free polaron; in complete analogy, we can derive the spectral properties of $H_{P}$ which are decisive to establish our final result.

The interaction between the three-dimensional polaron in a potential and the radiation field is described by the wellknown minimal substitution. Our method to calculate the local optical absorption spectrum of the system under consideration is based on a generalization of the spectral analysis performed in Ref. 11. The following conditions are to be fulfilled:

(i) The potential $V(r)$ violates completely the conservation of total momentum (see Refs. 12,13 for further comments and technical details).

(ii) The initial state of the polaron system is the ground state (low-temperature limit).

(iii) The phonon dispersion is taken in the form $\omega(q)=\omega$.

Additionally, we require that the incoming photon has a sharp momentum $q_{0}$ and a fixed polarization $\epsilon$.

It turns out that the local optical absorption spectrum $\Gamma(\Omega)$ can be expressed by a functional form of the ground state and a subset of excited states of the confined particle (the particle-phonon interaction included). In the following, we denote the corresponding energy eigenvalues and wave functions of the confined particle (the particle-phonon interaction included) by $E_{P}^{(m)}$ and $\left|\Phi_{P}^{(m)}\right\rangle$; in particular, $E_{P}^{(0)}$ and $\left|\Phi_{P}^{(0)}\right\rangle$ are ground-state energy and ground-state wave function, respectively. The quantum number $m$ contains the whole information about the corresponding eigenstate. The local optical absorption spectrum between bound-polaron states $\Gamma(\Omega)$ calculated in first-order time-dependent perturbation theory with respect to the electron-photon interaction is given by

$$
\Gamma \propto \frac{1}{\Omega} \sum_{(m, j) \neq(0,0)} \delta\left(E_{P}^{(m)}-E_{P}^{(0)}+j \hbar \omega-\hbar \Omega\right) \Pi_{m j} .
$$

Here, we have introduced the further abbreviations $\widetilde{G}_{q}$ $:=\left(v /(2 \pi)^{3}\right)\left|g_{q}\right|^{2}(v$ is the quantization volume) and

$$
\begin{aligned}
C_{m n}: & =\sum_{l} M_{q_{0} m l} \int d^{3} r \Psi_{l}^{*}(r) \epsilon p \Psi_{n}(r), \\
S_{m q_{1} \cdots q_{j}}= & \sum_{l n} C_{l n} \sum_{N=j}^{\infty} \prod_{\nu=j+1}^{N} \int d^{3} k_{\nu} \widetilde{G}_{k_{\nu}} e_{l k_{j+1} \cdots k_{N}}^{(m) *} \\
& \times \sum_{P\left(q_{1} \cdots q_{j} k_{j+1} \cdots k_{N}\right)} e_{n q_{1} \cdots q_{j} k_{j+1} \cdots k_{N}}^{(0)}, \\
\Pi_{m j}: & =\frac{1}{j !} \prod_{\nu=1}^{j} \int d^{3} q_{\nu} \widetilde{G}_{q_{\nu}}\left|S_{m q_{1} \cdots q_{j}}\right|^{2} .
\end{aligned}
$$

A detailed derivation of Eq. (6) as well as the generalization to the bound-exciton-phonon system will be given in a forthcoming publication. Direct inspection of Eq. (6) shows that $(1 / \Omega) \Pi_{m j}$ is a measure for the absorption strength of the corresponding transitions within the ladder of bound-polaron states. We emphasize that the existence of equidistant peaks separated by the LO-phonon energy is a general property under conditions (i)-(iii).

The presented model goes beyond previous theoretical work (either directly on polarons in quantum dots ${ }^{14}$ or applicable to bulk ${ }^{15}$ ) that is based on the adiabatic approximation. However, as has been clarified in the literature (for references, see Refs. 12,16,17), the adiabatic approximation for systems of Fröhlich type is applicable only in the strongcoupling regime ( $\alpha \gg 1)$, but is poor for weak and intermediate coupling strengths. A nonadiabatic treatment is presented in Ref. 16 and used to calculate the averaged absorption coefficient. We avoid here the adiabatic approximation using the functional-integral method. Our model is also useful for systems with close-lying confined energy states where next excited electronic states cannot be neglected. It also goes beyond the well-known Huang-Rhys model (see Ref. 18 and the extended version of Ref. 19). This model is a special case of ours: it neglects the nondiagonal part of $M_{q n_{1} n_{2}}$ in Eq. (1), thereby achieving analytical solvability. Some shortcomings of this approximation are pointed out in Ref. 8. The different point in our paper is that we use a rigorous result for the spectrum of the bound polaron, which is valid for a whole class of potentials $V$ (including Yukawa potentials and potentials of oscillator type): If $E$ is a spectral point of the Hamiltonian, the same is true for $E$ plus the LO-phonon energy. The most elegant proof of this property is provided by Feynman's functional-integral approach that shows that the exact partition function of the bound polaron has a prefactor, which is precisely the partition function of free phonons. A functional analytical proof is contained in Ref. 12. It is precisely this (and only this) property that we used to derive Eq. (6).

We have performed a model calculation for a parabolic, anisotropic potential of type $V(r) \sim \omega_{1}^{2} x_{1}^{2}+\omega_{2}^{2}\left(x_{2}^{2}+x_{3}^{2}\right)$, where $\omega_{i}$ is the potential strength in the corresponding space direction [the quantum numbers $m$ have the general structure $\left.m=\left(m_{1}, m_{2}, m_{3}\right)\right]$. An important result of our calculation is 


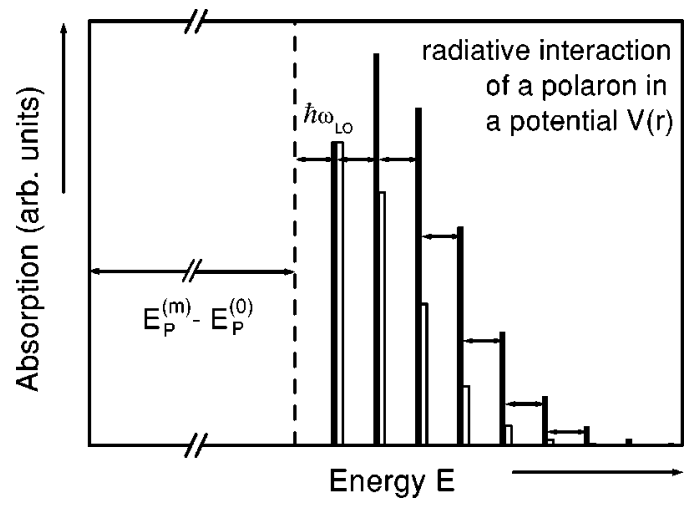

FIG. 3. Absorption strengths of excited states calculated for a bound polaron in a potential of type $V(r) \sim \omega_{1}^{2} x_{1}^{2}+\omega_{2}^{2}\left(x_{2}^{2}+x_{3}^{2}\right)$. Here, $E_{P}^{(m)}-E_{P}^{(0)}$ corresponds to the detection energy and is the difference between the energies of the ground state and the excited states with the quantum numbers $m=(0,1,0)$ and $m=(0,0,1)$ (see text for further explanations). The plotted curves correspond to the two cases $\omega_{1} \gg \omega_{2}$ (black) and $\omega_{1} \ll \omega_{2}$ (white).

the statement that anisotropies in the potential may strongly modify the optical transition probabilities. An example is illustrated in Fig. 3. The absorption strength is given for two examples with different anisotropies of the effective potential $V$. As can be seen, the obtained peak intensities are extremely sensitive with respect to potential anisotropies. Playing with the potential properties one can simulate both very different values for the maximum intensity and for the intensity slope down to zero. The deviation from the Poisson intensity distribution due to anisotropy can become much stronger than those reported as a result of nonadiabaticity. ${ }^{17}$ Such intensity variation should be observed, for example, in the infrared absorption for transitions to excited polaron states. An anisotropy in the potential is therefore one possi- bility to explain non-Poissonian intensity variation in the optical absorption. However, since we do not have access to the explicit wave functions in our calculation, line shapes and the contribution of the continuum absorption cannot be discussed here. For example, the resonance with continuum states might result in Fano effects or density of states enhancements due to free carrier absorption.

The technological realization of submonolayer growth now allows us to start with a detailed experimental study of bound polarons, addressing such problems as lifetime, dipole moment, binding energy, or Raman scattering involving bound-polaron states. Submonolayer growth, for example, is reported for both III-V and II-VI semiconductor quantum

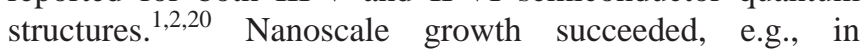
molecular-beam epitaxy of $\mathrm{CdSe}$ on $\mathrm{ZnSe}, \mathrm{ZnCdSe}$, or $\left.\mathrm{ZnMgSSe}^{21-26}\right)$. For quantum structures with a nominal layer thickness of CdSe between 0.5 and 1.0 ML, high efficient photoluminescence as well as optical gain has been observed with emission wavelengths in the blue/violet spectral range. But only little is known about the decoherence process, or the nonlinear optical properties of bound-polaron states. However, bound polarons have a fascinating particular property: the common phonon state is shared by all polarons. Such a property has a close analogy to atoms in traps sharing a common phonon state, a well-studied system in atomic physics. By growing semiconductor-based submonolayer structures in microcavities, we might exploit bound polarons coupled to a cavity photon state. The excited polaron states can then be used as spectator states for any measurement. Another interesting problem is the behavior under strong optical pumping above the band gap. Strong field-induced nonlinearities might be (coherently) transferred via the common phonon to another polaron state. Many other interesting phenomena are conceivable in semiconductor nanostructures based on the bound-polaron concept.
*Email address: woggon@ fred.physik.uni-dortmund.de

${ }^{1}$ I.L. Krestnikov, N. N. Ledentsov, A. Hoffmann, and D. Bimberg, Phys. Status Solidi A 183, 207 (2001).

${ }^{2}$ I.L. Krestnikov, M. Strassburg, M. Caesar, A. Hoffmann, U. W. Pohl, D. Bimberg, N. N. Ledentsov, P. S. Kopev, Zh. I. Alferov, D. Litvinov, A. Rosenauer, and D. Gerthsen, Phys. Rev. B 60, 8695 (1999).

${ }^{3}$ T. Passow, H. Heinke, D. Kayser, K. Leonardi, and D. Hommel, J. Cryst. Growth 214/215, 606 (2000).

${ }^{4}$ K.P. ODonnell and U. Woggon, Appl. Phys. Lett. 70, 2765 (1997).

${ }^{5}$ J. Conradi and R.R. Haering, Phys. Rev. Lett. 20, 1344 (1968).

${ }^{6}$ T. Itoh, M. Nishijima, A. I. Ekimov, C. Gourdon, AI. L. Efros, and M. Rosen, Phys. Rev. Lett. 74, 1645 (1995).

${ }^{7}$ S. Sauvage, P. Boucaud, R. P. S. M. Lobo, F. Bras, G. Fishman, R. Prazeres, F. Glotin, J. M. Ortega, and J. M. Gerard, Phys. Rev. Lett. 88, 177402 (2002).

${ }^{8}$ O. Verzelen, R. Ferreira, and G. Bastard, Phys. Rev. Lett. 88, 146803 (2002).

${ }^{9}$ F.M. Peeters, Wu. Xiaoguang, and J. T. Devreese, Phys. Rev. B 33, 3926 (1986).
${ }^{10}$ T. Mitra, A. Chatterjee, and S. Mukhopadhyay, Phys. Rep. 153, 91 (1987).

${ }^{11}$ B. Gerlach and F. Kalina, Phys. Rev. B 60, 10886 (1999).

${ }^{12}$ B. Gerlach and H. Löwen, Rev. Mod. Phys. 63, 63 (1991).

${ }^{13}$ H. Löwen, J. Math. Phys. 29, 1505 (1988).

${ }^{14}$ M.A. Odnoblyudov, I. N. Yassievich, and K. A. Chao, Phys. Rev. Lett. 83, 4884 (1999).

${ }^{15}$ S. Nakajima, Y. Toyozawa, and R. Abe, The Physics of Elementary Excitations (Springer, New York, 1980).

${ }^{16}$ V.M. Fomin, V. N. Gladilin, J. T. Devreese, E. P. Pokatilov, S. N. Balaban, and S. N. Klimin, Phys. Rev. B 57, 2415 (1998).

${ }^{17}$ J.T. Devreese, V. M. Fomin, V. N. Gladilin, E. P. Pokatilov, and S. N. Klimin, Nanotechnology 13, 163 (2002).

${ }^{18}$ K. Huang and A. Rhys, Proc. R. Soc. London, Ser. A 204, 406 (1950).

${ }^{19}$ C.B. Duke and G.D. Mahan, Phys. Rev. 139, A1965 (1965).

${ }^{20}$ N.N. Ledentsov, I. L. Krestnikov, M. V. Maximov, S. V. Ivanov, S. L. Sorokin, P. S. Kopev, Zh. I. Alferov, D. Bimberg, and C. M. Sotomayor-Torres, Appl. Phys. Lett. 69, 1343 (1996).

${ }^{21}$ Z.Q. Zhu, H. Yoshihara, K. Takebayashi, and T. Yao, Appl. Phys. Lett. 63, 1678 (1993). 
${ }^{22}$ D. Litvinov, A. Rosenauer, D. Gerthsen, and N. N. Ledentsov, Phys. Rev. B 61, 16819 (2000).

${ }^{23}$ F. Gindele, U. Woggon, W. Langbein, J. M. Hvam, K. Leonardi, D. Hommel, and H. Selke, Phys. Rev. B 60, 8773 (1999).

${ }^{24}$ K. Leonardi, H. Heinke, K. Ohkawa, D. Hommel, H. Selke, F. Gindele, and U. Woggon, Appl. Phys. Lett. 71, 1510 (1997).
${ }^{25}$ M. Strassburg, V. Kutzer, U. W. Pohl, A. Hoffmann, I. Broser, N. N. Ledentsov, D. Bimberg, A. Rosenauer, U. Fischer, D. Gerthsen, I. L. Krestnikov, M. V. Maximov, P. S. Kopev, and Zh. I. Alferov, Appl. Phys. Lett. 72, 942 (1998).

${ }^{26}$ S. Yamaguchi, Y. Kawakami, S. Fujita, S. Fujita, Y. Yamada, T. Mishina, and Y. Masumoto, Phys. Rev. B 54, 2629 (1996). 